\title{
WEIGHTED MINIMAL AND WEIGHTED FLAT SURFACES OF REVOLUTION IN GALILEAN 3-SPACE WITH DENSITY
}

\author{
AHMET KAZAN ${ }^{1, *}$ AND H. BAYRAM KARADAĞ ${ }^{2}$ \\ ${ }^{1}$ Department of Computer Technologies, Doğanşehir Vahap Küçük Vocational School of Higher Education, \\ Inonu University, Malatya, Turkey \\ ${ }^{2}$ Department of Mathematics, Faculty of Arts and Sciences, Inonu University, Malatya, Turkey \\ *Corresponding author: ahmet.kazan@inonu.edu.tr

\begin{abstract}
In this paper, we obtain the weighted mean and weighted Gaussian curvatures of surfaces of revolution in Galilean 3-space with density $e^{a_{1} x^{2}+a_{2} y^{2}+a_{3} z^{2}}, a_{1}, a_{2}, a_{3} \in \mathbb{R}$ not all zero. Also, we investigate some cases of weighted minimal surfaces of revolution according to $a_{i}, i=1,2,3$ and weighted flat surfaces of revolution.
\end{abstract}

\section{Introduction}

The geometry of surfaces of revolution is an important studying area for geometers and it has been studied widely in Euclidean 3-space $E^{3}$, Lorentz-Minkowski space $E_{1}^{3}$, Galilean 3-space $G_{3}$, pseudo-Galilean 3 -space $G_{3}^{1}$ and also in higher dimensions of these spaces. In these studies, the authors have investigated lots of characterizations about surfaces of revolution, but one of the most important characterization is minimal and flat surfaces of revolution. The catenoid which is obtained by rotating a catenary is the most famous minimal surface of revolution. For another characterizations of surfaces of revolution, we refer to [1], [3], [5], [6], [7], [8], [11], [12], [14] and etc.

2010 Mathematics Subject Classification. 53A10, 53A20, 53A35.

Key words and phrases. surface of revolution; isotropic rotation; weighted mean curvature; weighted gaussian curvature; weighted minimal and flat surface of revolution.

(C) 2018 Authors retain the copyrights of their papers, and all open access articles are distributed under the terms of the Creative Commons Attribution License. 
On the other hand, the geomerty of weighted manifold with density is a new studying area for geometers. In this sense, the weighted mean curvature $H_{\phi}$, which is also called $\phi$-mean curvature, of a surface in Euclidean 3 -space $E^{3}$ with density $e^{\phi}$ has been introduced in [9] and it is given by $H_{\phi}=H-\frac{1}{2}\langle N, \nabla \phi\rangle$, where $H$ is the mean curvature, $N$ is the unit normal vector of surface and $\nabla \phi$ is the gradient of $\phi$. The weighted mean curvature is a natural generalization of the mean curvature of a surface and a surface with $H_{\phi}=0$ is called a weighted minimal surface or a $\phi$-minimal surface.

Also in [2], the authors have introduced the notion of weighted Gaussian curvature or $\phi$-Gaussian curvature of a surface which is a generalization of the Gaussian curvature of a surface in a manifold with density $e^{\phi}$ and they have defined it as $G_{\phi}=G-\Delta \phi$. Here, $G$ is the Gaussian curvature of a surface and $\Delta$ is the Laplacian operator. If a surface's weighted Gaussian curvature is zero everywhere, then we call it a weighted flat surface or $\phi$-flat surface.

In [18], the translation surfaces in $G_{3}$ with a log-linear density have been studied and such a surface with vanishing weighted mean curvature has been classified. In [13], the authors have considered the Euclidean 3 -space $E^{3}$ with a positive density function $e^{\phi}$, where $\phi=-x^{2}-y^{2},(x, y, z) \in E^{3}$ and they have constructed all the helicoidal surfaces in the space by solving the second-order non-linear ordinary differential equation with the weighted Gaussian curvature and the mean curvature functions. Also in [10], the authors have studied the ruled surfaces and translation surfaces in $E^{3}$ with density $e^{z}$ and as a generalization of this density, Lopez has used a linear density $e^{a x+b y+c z}, a, b, c \in \mathbb{R}$ and classified weighted minimal translation and cyclic surfaces in $E^{3}[15]$.

In the present paper, we obtain the weighted mean curvature and weighted Gaussian curvature of three types of surfaces of revolution in Galilean 3 -space with density $e^{a_{1} x^{2}+a_{2} y^{2}+a_{3} z^{2}}, a_{i} \in \mathbb{R}$ not all zero and investigate some cases of weighted minimal surfaces of revolution according to $a_{i}, i=1,2,3$ and weighted flat surfaces of revolution. Also, we draw the obtaining surfaces of revolution with the aid of Mathematica.

\section{Preliminaries}

The Galilean space $G_{3}$ is a Cayley-Klein space equipped with the projective metric of signature $(0,0,+,+)$. The absolute figure of the Galilean geometry consists of an ordered triple $\{w, f, I\}$, where $w$ is the ideal (absolute) plane, $f$ the line (absolute line) in $w$ and $I$ the fixed elliptic involution of points of $p$.

A vector $\mathbf{x}=\left(x_{1}, x_{2}, x_{3}\right)$ in $G_{3}$ is isotropic if $x_{1}=0$ and non-isotropic otherwise. So, for affine ccordinates $(x, y, z)$, the $y$-axis and $z$-axis are isotropic while the $x$-axis is non-isotropic. Also, the $y z$-plane is Euclidean and the $x y$-plane and $x z$-plane are isotropic. If $\mathbf{x}=\left(x_{1}, x_{2}, x_{3}\right)$ and $\mathbf{y}=\left(y_{1}, y_{2}, y_{3}\right)$ are two vectors in $G_{3}$, then the Galilean scalar product of $\mathbf{x}$ and $\mathbf{y}$ is defined by

$$
\langle\mathbf{x}, \mathbf{y}\rangle=\left\{\begin{array}{cl}
x_{1} y_{1}, & \text { if } x_{1} \neq 0 \text { or } y_{1} \neq 0 \\
x_{2} y_{2}+x_{3} y_{3}, & \text { if } x_{1}=0 \text { and } y_{1}=0
\end{array}\right.
$$


So, the Galilean norm of a vector $\mathbf{x}$ in $G_{3}$ is $\|\mathbf{x}\|=\sqrt{\langle\mathbf{x}, \mathbf{x}\rangle}$ and the vector $\mathbf{x}$ is a unit vector if $\|\mathbf{x}\|=1$. The Galilean cross product of $\mathbf{x}$ and $\mathbf{y}$ is defined by

$$
\mathbf{x} \times \mathbf{y}=\left|\begin{array}{ccc}
0 & e_{2} & e_{3} \\
x_{1} & x_{2} & x_{3} \\
y_{1} & y_{2} & y_{3}
\end{array}\right| .
$$

For a more detailed treatment about Galilean space, we refer to [3], [4], [16], [17], [18] and etc.

Furthermore, let $M$ be a surface in $G_{3}$ parametrized by

$$
\Gamma\left(u^{1}, u^{2}\right)=\left(x\left(u^{1}, u^{2}\right), y\left(u^{1}, u^{2}\right), z\left(u^{1}, u^{2}\right)\right) .
$$

Then, the unit normal vector $N$ of the surface is defined by

$$
N=\frac{\Gamma_{, 1} \times \Gamma_{, 2}}{w}
$$

where $w=\left\|\Gamma_{, 1} \times \Gamma_{, 2}\right\|$ and $\Gamma_{, i}=\frac{\partial \Gamma}{\partial u^{i}}\left(u^{1}, u^{2}\right)$ for $i \in\{1,2\}$. The coefficients of the second fundamental form are given by

$$
L_{i j}=\left\langle\frac{\Gamma_{, i j} x_{, 1}-x_{, i j} \Gamma_{, 1}}{x_{, 1}}, N\right\rangle=\left\langle\frac{\Gamma_{, i j} x_{, 2}-x_{, i j} \Gamma_{, 2}}{x_{, 2}}, N\right\rangle
$$

Analogous to Euclidean space, in [16], the mean curvature $H$ and the Gaussian curvature $K$ of the surface are defined by

$$
H=\frac{1}{2} \sum_{i, j=1}^{2} g^{i j} L_{i j} \quad \text { and } \quad K=\frac{L_{11} L_{22}-L_{12}^{2}}{w^{2}},
$$

where $g^{i j}=g^{i} g^{j}$, for $i, j \in\{1,2\}$ and $g^{1}=\frac{x_{, 2}}{w}, g^{2}=-\frac{x_{, 1}}{w}$.

Here we always assume that the surface is admissible, that is, its tangent space is nowhere an Euclidean plane (for detail, see [3]).

\section{Weighted Minimal and Weighted Flat Surfaces of Revolution in $G_{3}$ with Density $e^{a_{1} x^{2}+a_{2} y^{2}+a_{3} z^{2}}$}

In [3], the authors have constructed the surfaces of revolution in Galilean 3-space analogously to how that is done in Euclidean 3-space and they have obtained 3 types of surfaces of revolution in $G_{3}$.

Since there are different kinds of planes in $G_{3}$, we have to consider two possibilities for the supporting plane of the profile curve which generates the surface of revolution. In this context, the profile curve lies in an Euclidean plane or it lies in an isotropic plane.

In order to construct a surface of revolution in $G_{3}$, we use two types of rotations which are defined as follows: 
An Euclidean rotation about the non-isotropic $x$-axis is given by

$$
\left[\begin{array}{l}
x^{\prime} \\
y^{\prime} \\
z^{\prime}
\end{array}\right]=\left[\begin{array}{ccc}
1 & 0 & 0 \\
0 & \cos \theta_{1} & \sin \theta_{1} \\
0 & -\sin \theta_{1} & \cos \theta_{1}
\end{array}\right]\left[\begin{array}{l}
x \\
y \\
z
\end{array}\right],
$$

where $\theta_{1}$ is the Euclidean angle and an isotropic rotation is given by

$$
\left[\begin{array}{c}
x^{\prime} \\
y^{\prime} \\
z^{\prime}
\end{array}\right]=\left[\begin{array}{ccc}
1 & 0 & 0 \\
\theta_{2} & 1 & 0 \\
0 & 0 & 1
\end{array}\right]\left[\begin{array}{l}
x \\
y \\
z
\end{array}\right]+\left[\begin{array}{c}
c \theta_{2} \\
\frac{c}{2}\left(\theta_{2}\right)^{2} \\
0
\end{array}\right],
$$

where $\theta_{2}$ is the isotropic angle and $c \in \mathbb{R}$.

\subsection{Weighted Minimal and Weighted Flat Type I Surfaces of Revolution in $G_{3}$.}

Type I surface of revolution has constructed with the aid of an isotropic rotation as

$$
\Gamma_{I}(u, v)=\left(c v, f(u)+\frac{c}{2} v^{2}, g(u)\right)
$$

where the profile curve $\alpha$ lies in the Euclidean $y z$-plane and it is parametrized by $\alpha(u)=(0, f(u), g(u))[3]$. Here, $f$ and $g$ are real functions and we assume that, the profile curve $\alpha$ is given by the arc length, that is

$$
f^{\prime 2}(u)+g^{\prime 2}(u)=1, \forall u \in I
$$

Using (3.2), we obtain the mean curvature and Gaussian curvature of (3.1) as

$$
H=\frac{f^{\prime \prime}(u) g^{\prime}(u)-f^{\prime}(u) g^{\prime \prime}(u)}{2} \text { and } K=\frac{f^{\prime \prime}(u)}{c}
$$

respectively. On the other hand, the unit normal vector $N$ of the surface (3.1) is

$$
N=\left(0, g^{\prime}(u),-f^{\prime}(u)\right)
$$

Assume that, $M$ is the surface in $G_{3}$ with density $e^{\phi}, \phi=a_{1} x^{2}+a_{2} y^{2}+a_{3} z^{2}, a_{1}, a_{2}, a_{3} \in \mathbb{R}$ not all zero. Then, for this density, from (3.1)-(3.4) the weighted mean curvature and the weighted Gaussian curvature can be obtained as

$$
H_{\phi}=\frac{f^{\prime \prime} g^{\prime}-f^{\prime} g^{\prime \prime}}{2}-<\left(a_{1} c v, a_{2}\left(f+\frac{c}{2} v^{2}\right), a_{3} g\right),\left(0, g^{\prime},-f^{\prime}\right)>
$$

and

$$
K_{\phi}=\frac{f^{\prime \prime}}{c}-2\left(a_{1}+a_{2}+a_{3}\right)
$$

respectively.

Firstly, let us assume that the surface of revolution (3.1) is weighted minimal, i.e., $H_{\phi}=0$. Then, from (3.5) we have

$$
f^{\prime \prime} g^{\prime}-f^{\prime} g^{\prime \prime}-2<\left(a_{1} c v, a_{2}\left(f+\frac{c}{2} v^{2}\right), a_{3} g\right),\left(0, g^{\prime},-f^{\prime}\right)>=0 .
$$

Now, we'll investigate some cases of weighted minimal surfaces of revolution $(3.1)$ according to $a_{i}, i=1,2,3$. 
If $a_{1} \neq 0$, then from (3.7) we get

$$
f^{\prime \prime} g^{\prime}-f^{\prime} g^{\prime \prime}=0
$$

Here, using (3.2) in (3.8) we obtain

$$
f(u)=c_{1} u+c_{2}
$$

and using (3.9) in (3.2) we get

$$
g(u)=\sqrt{1-c_{1}^{2}} u+c_{3},
$$

where $c_{1}, c_{2}, c_{3} \in \mathbb{R}$. Hence, we have

Theorem 3.1. Let $M$ be a weighted minimal type I surface of revolution in Galilean 3-space with density $e^{a_{1} x^{2}+a_{2} y^{2}+a_{3} z^{2}}$, where $a_{1} \neq 0, a_{2}$ and $a_{3}$ are constants. Then, $M$ can be parametrized by

$$
\Gamma_{I}(u, v)=\left(c v, c_{1} u+c_{2}+\frac{c}{2} v^{2}, \sqrt{1-c_{1}^{2}} u+c_{3}\right),
$$

for some constants $c, c_{1}, c_{2}$ and $c_{3}$.

In Figure 1, one can see the surface of revolution (3.11) for $c=4, c_{1}=1 / 2, c_{2}=2$ and $c_{3}=3$.

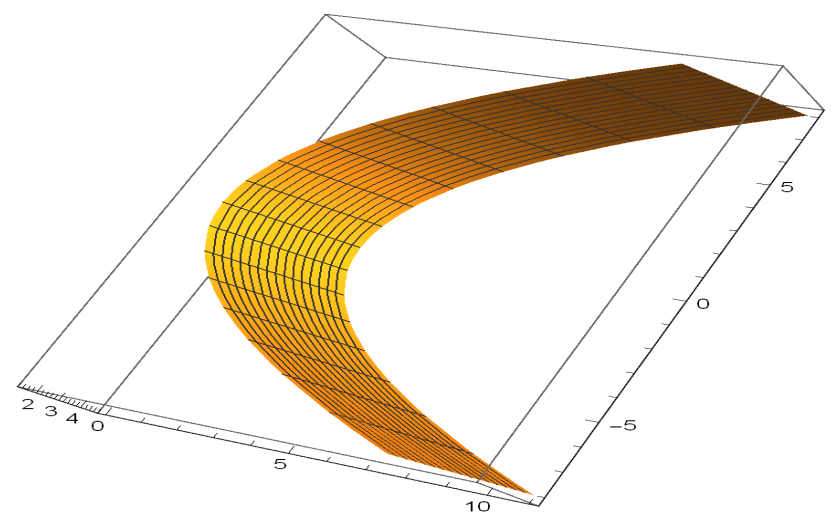

FiguRe 1.

If $a_{1}=0$, then from (3.7) we get

$$
f^{\prime \prime} g^{\prime}-f^{\prime} g^{\prime \prime}-2\left(a_{2}\left(f g^{\prime}+\frac{c}{2} v^{2} g^{\prime}\right)-a_{3} f^{\prime} g\right)=0 .
$$

Taking $a_{2}=0$ in (3.12) and using (3.2), we get

$$
g^{\prime \prime}-2 a_{3} g\left(1-\left(g^{\prime}\right)^{2}\right)=0
$$

The equation (3.13) is a second-order nonlinear ordinary differential equation and $g(u)=u+c_{4}, c_{4} \in \mathbb{R}$, is a special solution for it. So, for this special solution, from (3.2) we get $f(u)=c_{5}, c_{5} \in \mathbb{R}$. Hence, we have 
Theorem 3.2. Let $M$ be a weighted minimal type I surface of revolution in Galilean 3-space with density $e^{a_{3} z^{2}}$, where $a_{3}$ is a non-zero constant. Then, $M$ can be parametrized by

$$
\Gamma_{I}(u, v)=\left(c v, c_{5}+\frac{c}{2} v^{2}, u+c_{4}\right)
$$

for some constants $c, c_{4}$ and $c_{5}$.

In Figure 2, one can see the surface of revolution (3.14) for $c=1, c_{4}=2$ and $c_{5}=3$.

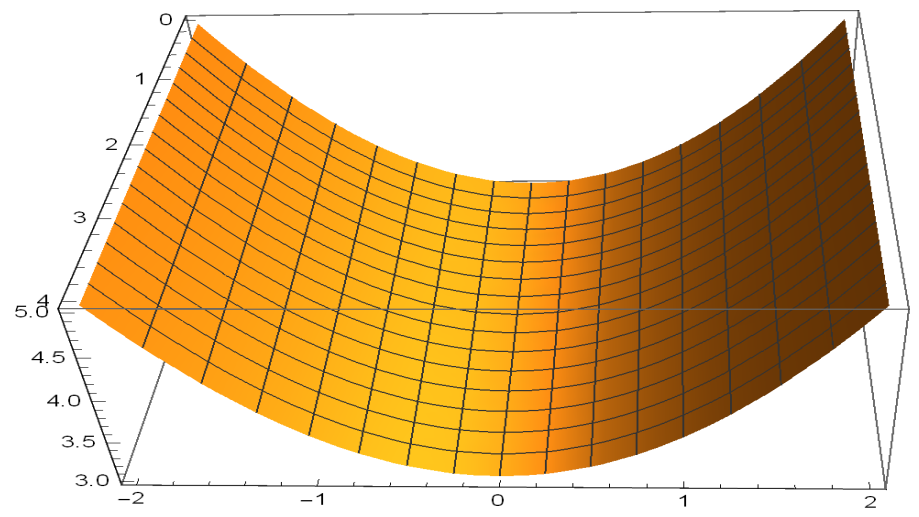

FIGURE 2.

Now, let us investigate the weighted flat surfaces of revolution (3.1). If the surface of revolution (3.1) is weighted flat, i.e., $K_{\phi}=0$, then from (3.6) we have

$$
f^{\prime \prime}-2 c\left(a_{1}+a_{2}+a_{3}\right)=0
$$

From (3.15), we have

$$
f(u)=c r u^{2}+c_{6} u+c_{7}
$$

and using (3.16) in (3.2), we have

$$
g(u)=\mp \frac{1}{4 c r}\left(\left(c_{6}+2 c r u\right) \sqrt{1-c_{6}^{2}-4 c c_{6} r u-4 c^{2} r^{2} u^{2}}+\arcsin \left(c_{6}+2 c r u\right)\right)+c_{8},
$$

where $r=a_{1}+a_{2}+a_{3}$ and $c, c_{6}, c_{7}, c_{8}$ are constants. Hence, we have

Theorem 3.3. Let $M$ be a weighted flat type I surface of revolution in Galilean 3-space with density $e^{a_{1} x^{2}+a_{2} y^{2}+a_{3} z^{2}}, a_{1}, a_{2}, a_{3} \in \mathbb{R}$ not all zero. Then, $M$ can be parametrized by

$$
\begin{aligned}
\Gamma_{I}(u, v) & =\left(c v, c r u^{2}+c_{6} u+c_{7}+\frac{c}{2} v^{2},\right. \\
& \left.\mp \frac{1}{4 c r}\left(\left(c_{6}+2 c r u\right) \sqrt{1-c_{6}^{2}-4 c c_{6} r u-4 c^{2} r^{2} u^{2}}+\arcsin \left(c_{6}+2 c r u\right)\right)+c_{8}\right),
\end{aligned}
$$

for some constants $c, c_{6}, c_{7}, c_{8}$ and $r=a_{1}+a_{2}+a_{3}$. 
Figure 3 shows the weighted flat type I surface of revolution $(3.18)$ for $r=6, c=4, c_{6}=1, c_{7}=2$ and $c_{8}=3$.

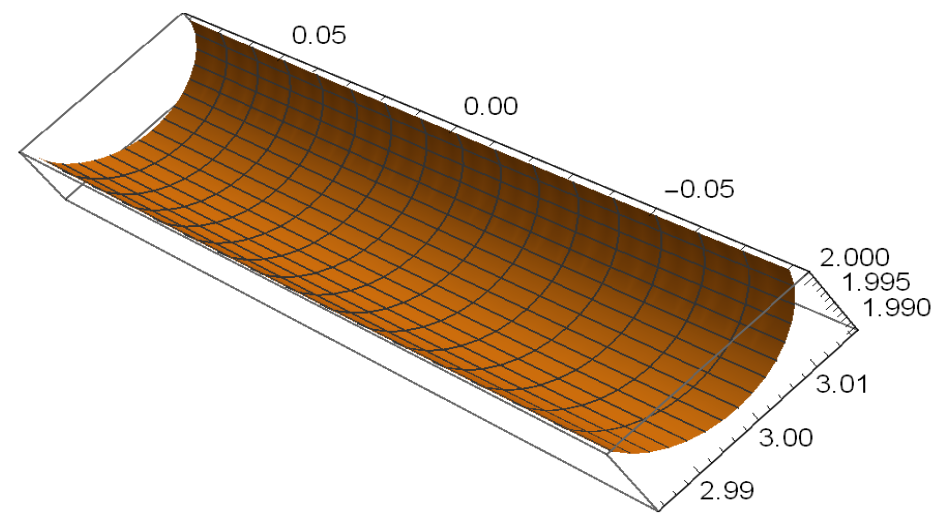

FiguRE 3.

\subsection{Weighted Minimal and Weighted Flat Type II Surfaces of Revolution in $G_{3}$.}

Type II surface of revolution can be obtained with the aid of an isotropic rotation as

$$
\Gamma_{I I}(u, v)=\left(u+c v, g(u), u v+\frac{c}{2} v^{2}\right)
$$

where the profile curve $\alpha$ lies in the isotropic $x y$-plane and it is parametrized by $\alpha(u)=(f(u), g(u), 0)[3]$. Here, $f$ and $g$ are real functions and we assume that, the profile curve $\alpha$ is given by the arc length, that is

$$
f^{\prime 2}(u)=1, \forall u \in I
$$

We obtain the mean curvature and Gaussian curvature of (3.19) as

$$
H=\frac{-c^{2}\left(u g^{\prime \prime}(u)-g^{\prime}(u)\right)}{2\left(u^{2}+c^{2}\left(g^{\prime}(u)\right)^{2}\right)^{\frac{3}{2}}} \text { and } K=\frac{c^{2} g^{\prime}(u)\left(u g^{\prime \prime}(u)-g^{\prime}(u)\right)}{\left(u^{2}+c^{2}\left(g^{\prime}(u)\right)^{2}\right)^{2}},
$$

respectively. On the other hand, the unit normal vector $N$ of the surface (3.19) is

$$
N=\frac{1}{\sqrt{u^{2}+c^{2}\left(g^{\prime}(u)\right)^{2}}}\left(0,-u,-c g^{\prime}(u)\right)
$$

Assume that, $M$ is the surface in $G_{3}$ with density $e^{\phi}, \phi=a_{1} x^{2}+a_{2} y^{2}+a_{3} z^{2}$. Then, for this density, from (3.19)-(3.22) the weighted mean curvature and the weighted Gaussian curvature can be obtained as

$$
H_{\phi}=\frac{-c^{2}\left(u g^{\prime \prime}-g^{\prime}\right)}{2\left(u^{2}+c^{2}\left(g^{\prime}\right)^{2}\right)^{\frac{3}{2}}}-\frac{1}{\sqrt{u^{2}+c^{2}\left(g^{\prime}\right)^{2}}}<\left(a_{1}(u+c v), a_{2} g, a_{3}\left(u v+\frac{c}{2} v^{2}\right)\right),\left(0,-u,-c g^{\prime}\right)>
$$

and

$$
K_{\phi}=\frac{c^{2} g^{\prime}\left(u g^{\prime \prime}-g^{\prime}\right)}{\left(u^{2}+c^{2}\left(g^{\prime}\right)^{2}\right)^{2}}-2\left(a_{1}+a_{2}+a_{3}\right)
$$

respectively. 
Firstly, let us assume that the surface of revolution (3.19) is weighted minimal, i.e., $H_{\phi}=0$. Then, from (3.23) we have

$$
c^{2}\left(u g^{\prime \prime}-g^{\prime}\right)+2\left(u^{2}+c^{2}\left(g^{\prime}\right)^{2}\right)<\left(a_{1}(u+c v), a_{2} g, a_{3}\left(u v+\frac{c}{2} v^{2}\right)\right),\left(0,-u,-c g^{\prime}\right)>=0 .
$$

Now, we'll investigate some cases of weighted minimal surfaces of revolution (3.19) according to $a_{i}, i=1,2,3$.

If $a_{1} \neq 0$, then from $(3.25)$ we get

$$
c^{2}\left(u g^{\prime \prime}-g^{\prime}\right)=0
$$

So, from (3.26) we get

$$
g(u)=\frac{c_{1}}{2} u^{2}+c_{2}
$$

where $c_{1}, c_{2} \in \mathbb{R}$. Hence, we have

Theorem 3.4. Let $M$ be a weighted minimal type II surface of revolution in Galilean 3-space with density $e^{a_{1} x^{2}+a_{2} y^{2}+a_{3} z^{2}}$, where $a_{1} \neq 0, a_{2}$ and $a_{3}$ are constants. Then, $M$ can be parametrized by

$$
\Gamma_{I I}(u, v)=\left(u+c v, \frac{c_{1}}{2} u^{2}+c_{2}, u v+\frac{c}{2} v^{2}\right)
$$

for some constants $c, c_{1}$ and $c_{2}$.

The shape of the surface of revolution (3.28) for $c=3, c_{1}=1$ and $c_{2}=2$ can be seen in Figure 4 as follows:

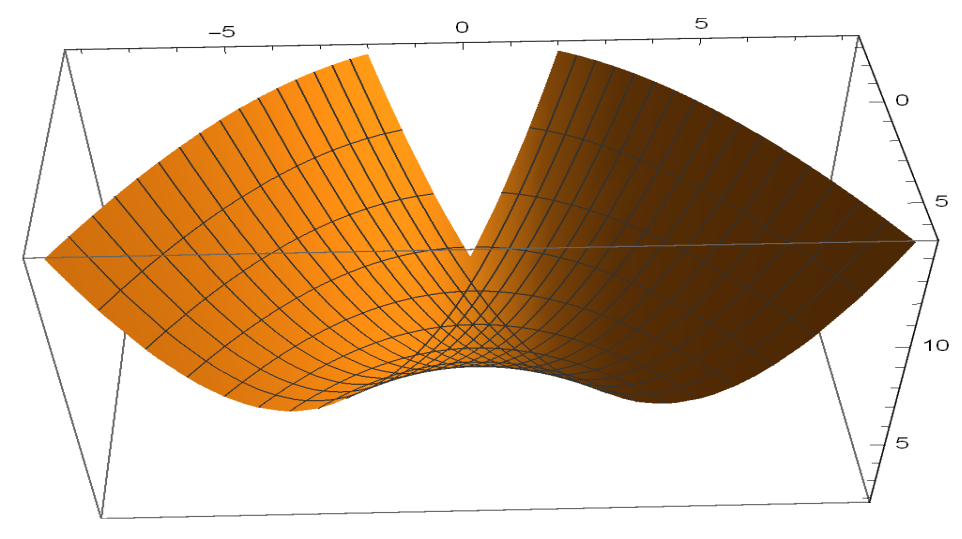

Figure 4.

If $a_{1}=0$, then from (3.25) we get

$$
c^{2}\left(u g^{\prime \prime}-g^{\prime}\right)+2\left(u^{2}+c^{2}\left(g^{\prime}\right)^{2}\right)\left(-a_{2} u g-a_{3} c g^{\prime}\left(u v+\frac{c}{2} v^{2}\right)\right)=0 .
$$

Taking $a_{3}=0$ in (3.29), we get

$$
c^{2}\left(u g^{\prime \prime}-g^{\prime}\right)-2 a_{2} u g\left(u^{2}+c^{2}\left(g^{\prime}\right)^{2}\right)=0 .
$$

Hence, we have 
Theorem 3.5. Let $M$ be a weighted flat type II surface of revolution in Galilean 3-space with density $e^{a_{2} y^{2}}$, where $a_{2}$ is a non-zero constant. Then, the real function $g$ which is in (3.19) must satisfy the second-order nonlinear ordinary differential equation (3.30).

Now, let us investigate the weighted flat surfaces of revolution (3.19). If the surface of revolution (3.19) is weighted flat, i.e., $K_{\phi}=0$, then from (3.24) we have

$$
c^{2} g^{\prime}\left(u g^{\prime \prime}-g^{\prime}\right)-2 r\left(u^{2}+c^{2}\left(g^{\prime}\right)^{2}\right)^{2}=0,
$$

where $r=a_{1}+a_{2}+a_{3}$. From (3.31), we have

$$
c^{2} u g^{\prime} g^{\prime \prime}-c^{2} g^{\prime 2}-2 r u^{4}-4 r u^{2} c^{2} g^{\prime 2}-2 r c^{4} g^{\prime 4}=0 .
$$

Taking $p(u)=g^{\prime}(u)$ in (3.32), we get

$$
p(u)=\mp \frac{\sqrt{-2 r u^{4}-u^{2}-2 c^{2} c_{4} u^{2}}}{\sqrt{2} \sqrt{c^{2} r u^{2}+c^{4} c_{4}}}
$$

and integrating (3.33), we have

$$
g(u)= \pm \frac{u \sqrt{2 r u^{2}+2 c^{2} c_{4}+1}\left(\begin{array}{c}
\sqrt{2} \arcsin h\left(\sqrt{2} \sqrt{r u^{2}+c^{2} c_{4}}\right) \sqrt{r u^{2}+c^{2} c_{4}} \\
+2\left(r u^{2}+c^{2} c_{4}\right) \sqrt{2 r u^{2}+2 c^{2} c_{4}+1}
\end{array}\right)}{4 \sqrt{2} r \sqrt{-u^{2}\left(2 r u^{2}+2 c^{2} c_{4}+1\right)} \sqrt{c^{2} r u^{2}+c^{4} c_{4}}},
$$

where $r=a_{1}+a_{2}+a_{3}, c$ and $c_{4}$ are constants. Hence, we have

Theorem 3.6. Let $M$ be a weighted flat type II surface of revolution in Galilean 3-space with density $e^{a_{1} x^{2}+a_{2} y^{2}+a_{3} z^{2}}, a_{1}, a_{2}, a_{3} \in \mathbb{R}$ not all zero. Then, $M$ can be parametrized by

$$
\begin{aligned}
& \Gamma_{I}(u, v)=(u+c v, \\
& \pm \frac{u \sqrt{2 r u^{2}+2 c^{2} c_{4}+1}\left(\begin{array}{c}
\sqrt{2} \arcsin h\left(\sqrt{2} \sqrt{r u^{2}+c^{2} c_{4}}\right) \sqrt{r u^{2}+c^{2} c_{4}} \\
+2\left(r u^{2}+c^{2} c_{4}\right) \sqrt{2 r u^{2}+2 c^{2} c_{4}+1}
\end{array}\right)}{4 \sqrt{2} r \sqrt{-u^{2}\left(2 r u^{2}+2 c^{2} c_{4}+1\right)} \sqrt{c^{2} r u^{2}+c^{4} c_{4}}}, \\
&\left.u v+\frac{c}{2} v^{2}\right),
\end{aligned}
$$

for some constants $c, c_{4}$ and $r=a_{1}+a_{2}+a_{3}$.

Figure 5 shows the weighted flat type II surface of revolution (3.35) for $r=-6, c=2$ and $c_{4}=1$. 


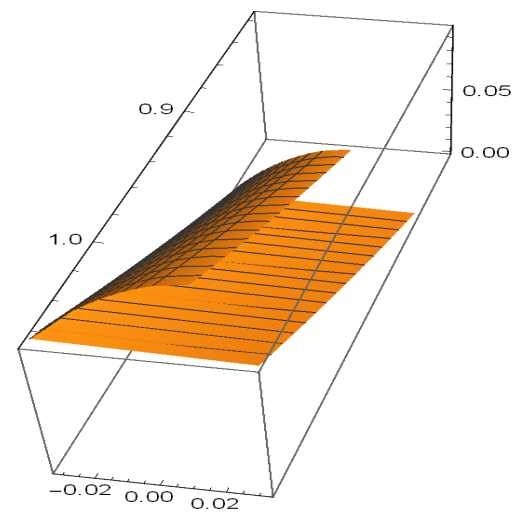

FIGURE 5.

\subsection{Weighted Minimal and Weighted Flat Type III Surfaces of Revolution in $G_{3}$.}

Type III surface of revolution can be obtained with the aid of an Euclidean rotation about the axis $x$ as

$$
\Gamma_{I I I}(u, v)=(u, g(u) \cos v,-g(u) \sin v)
$$

where the profile curve $\alpha$ lies in the isotropic $x y$-plane and it is parametrized by $\alpha(u)=(f(u), g(u), 0)[3]$. Here, $f$ and $g$ are real functions and we assume that, the profile curve $\alpha$ is given by the arc length, that is

$$
f^{\prime 2}(u)=1, \forall u \in I
$$

Now, we obtain the mean curvature and Gaussian curvature of the surface of revolution (3.36) as

$$
H=-\frac{1}{2 g(u)} \text { and } K=-\frac{g^{\prime \prime}(u)}{g(u)},
$$

respectively. On the other hand, the unit normal vector $N$ of the surface (3.36) is

$$
N=(0, \cos v,-\sin v) .
$$

Assume that, $M$ is the surface in $G_{3}$ with density $e^{\phi}, \phi=a_{1} x^{2}+a_{2} y^{2}+a_{3} z^{2}$. Then, for this density, from (3.36)-(3.39) the weighted mean curvature and the weighted Gaussian curvature can be obtained as

$$
H_{\phi}=-\frac{1}{2 g}-<\left(a_{1} u, a_{2} g \cos v,-a_{3} g \sin v\right),(0, \cos v,-\sin v)>
$$

and

$$
K_{\phi}=-\frac{g^{\prime \prime}(u)}{g(u)}-2\left(a_{1}+a_{2}+a_{3}\right)
$$

respectively.

Firstly, let us assume that the surface of revolution (3.36) is weighted minimal, i.e., $H_{\phi}=0$. Then, from (3.40) we have

$$
\frac{1}{2 g}+<\left(a_{1} u, a_{2} g \cos v,-a_{3} g \sin v\right),(0, \cos v,-\sin v)>=0
$$

Next, we'll investigate some cases of weighted minimal surfaces of revolution (3.36) according to $a_{i}, i=1,2,3$. 
If $a_{1} \neq 0$, then from (3.42) we get

$$
\frac{1}{2 g}=0
$$

and this is a contradiction. Hence, we have

Theorem 3.7. There is no weighted minimal type III surface of revolution in Galilean 3-space with density $e^{a_{1} x^{2}+a_{2} y^{2}+a_{3} z^{2}}$, where $a_{1} \neq 0, a_{2}$ and $a_{3}$ are constants.

If $a_{1}=0$, then from (3.42) we get

$$
\frac{1}{2 g}+a_{2} g \cos ^{2} v+a_{3} g \sin ^{2} v=0
$$

Taking $a_{2}=a_{3}=\mathrm{a} \neq 0$ in (3.44), we get

$$
\frac{1}{2 g}+\mathrm{a} g=0
$$

and so,

$$
g(u)=\mp \sqrt{-\frac{1}{2 \mathrm{a}}}, \mathrm{a}<0 .
$$

Hence, we have

Theorem 3.8. Let $M$ be a weighted minimal type III surface of revolution in Galilean 3-space with density $e^{\mathrm{a}\left(y^{2}+z^{2}\right)}$, where $\mathrm{a}<0$ is a constant. Then, $M$ can be parametrized by

$$
\Gamma_{I I I}(u, v)=\left(u, \mp \sqrt{-\frac{1}{2 \mathrm{a}}} \cos v, \pm \sqrt{-\frac{1}{2 \mathrm{a}}} \sin v\right) .
$$

The shape of the surface of revolution (3.47) for $\mathrm{a}=-1$ is given in Figure 6 .

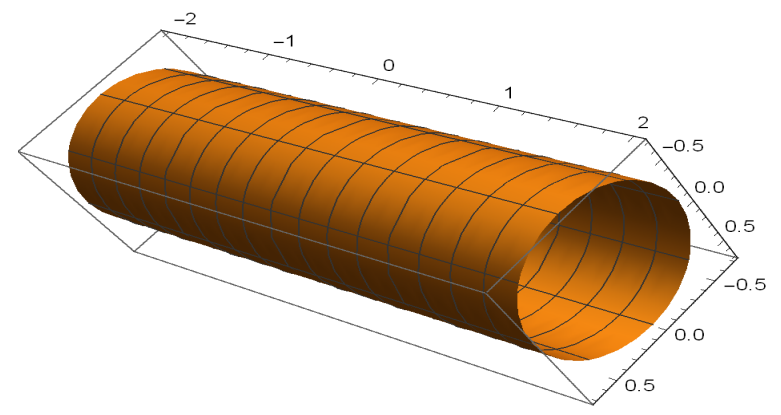

FiguRe 6.

Now, let us investigate the weighted flat surfaces of revolution (3.36). If the surface of revolution (3.36) is weighted flat, i.e., $K_{\phi}=0$, then from (3.41) we have

$$
\frac{g^{\prime \prime}}{g}+2 r=0
$$


where $r=a_{1}+a_{2}+a_{3}$. From (3.48), we have

$$
g(u)=c_{1} \cos (\sqrt{2 r} u)+c_{2} \sin (\sqrt{2 r} u)
$$

Hence, we have

Theorem 3.9. Let $M$ be a weighted flat type III surface of revolution in Galilean 3-space with density $e^{a_{1} x^{2}+a_{2} y^{2}+a_{3} z^{2}}, a_{1}, a_{2}, a_{3} \in \mathbb{R}$ not all zero. Then, $M$ can be parametrized by

$$
\Gamma_{I I I}(u, v)=\left(u,\left(c_{1} \cos (\sqrt{2 r} u)+c_{2} \sin (\sqrt{2 r} u)\right) \cos v,-\left(c_{1} \cos (\sqrt{2 r} u)+c_{2} \sin (\sqrt{2 r} u)\right) \sin v\right)
$$

for some constants $c_{1}, c_{2}$ and $r=a_{1}+a_{2}+a_{3}$.

Figure 7 shows the weighted flat type III surface of revolution (3.50) for $r=2, c_{1}=1$ and $c_{2}=3$.

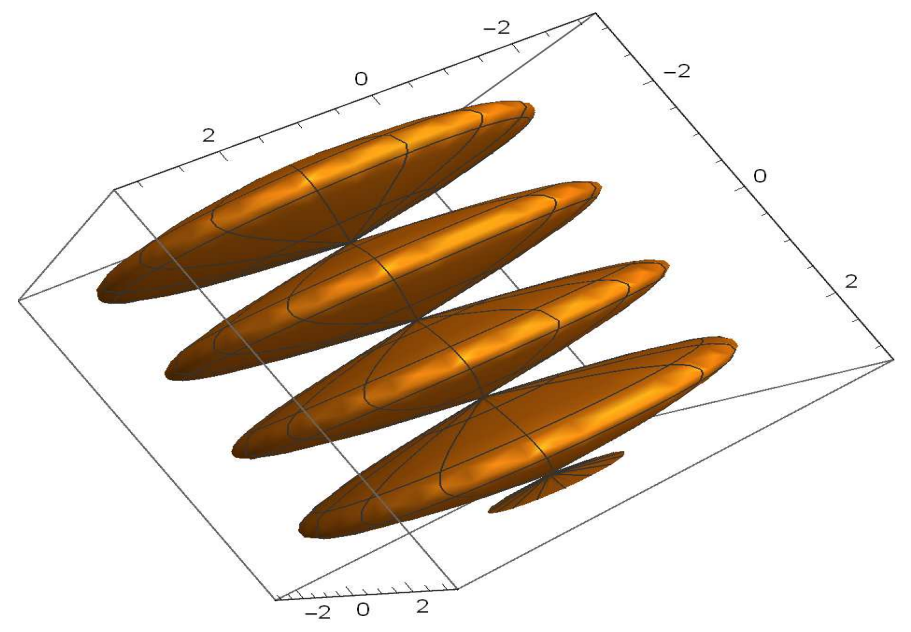

FiguRE 7.

\section{REFERENCES}

[1] M. Bekkar and H. Zoubir, Surfaces of revolution in the 3-dimensional Lorentz-Minkowski space satisfying $\Delta x^{i}=\lambda^{i} x^{i}$, Int. J. Contemp. Math. Sci. 24 (3) (2008), 1173-1185.

[2] I. Corwin, H. Hoffman, S. Hurder, V. Ssum and Y. Xu, Differential geometry of manifolds with density, Rose-Hulman Und. Math. J. 7 (2006), 1-15.

[3] M. Dede, C. Ekici and W. Goemans, Surfaces of Revolution with Vanishing Curvature in Galilean 3-Space, J. Math. Phys. in press.

[4] M. Dede, Tubular Surfaces in Galilean Space, Math. Commun. 18 (2013), 209-217. 
[5] F. Dillen, J. Pas and L. Verstraelen, On the Gauss map of surfaces of revolution, Bull. Inst. Math. Acad. Sinica 18 (1990), 239-246.

[6] B. Divjak and Z.M. Sipus, Some Special Surfaces in the Pseudo-Galilean Space, Acta Math. Hungar. 118 (3) (2008), 209-226.

[7] A. Ferrandez and P. Lucas, On Surfaces in the 3-dimensional Lorentz-Minkowski space, Pac. J. Math. 152 (1) (1992), 93-100.

[8] O.J. Garay, On a certain class of finite type surfaces of revolution, Kodai Math. J. 11 (1) (1988), 25-31.

[9] M. Gromov, Isoperimetry of waists and concentration of maps, Geom. Func. Anal. 13 (2003), 178-215.

[10] D.T. Hieu and N.M Hoang, Ruled minimal surfaces in $\mathbb{R}^{3}$ with density $e^{z}$, Pac. J. Math. 243 (2009), 277-285.

[11] G. Kaimakamis and B. Papantoniou, Surfaces of revolution in the 3-dimensional Lorentz-Minkowski space satisfying $\Delta^{I I} \vec{r}=$ $A \vec{r}$, J. Geom. 81 (2004), 81-92.

[12] A. Kazan and H.B. Karadağ, A Classification of Surfaces of Revolution in Lorentz-Minkowski Space, Int. J. Contemp. Math. Sci. 39 (6) (2011), 1915-1928.

[13] D-S. Kim and D.W. Yoon, Constructions of Helicoidal Surfaces in Euclidean Space with Density, Symmetry 9 (2017), Art. ID 173.

[14] S. Lee and J.H. Varnado, Spacelike constant mean curvature surfaces of revolution in Minkowski 3-space, Differ. Geom. Dyn. Syst. 8 (1) (2006), 144-165.

[15] R. López, Minimal surface in Euclidean Space with a Log-Linear Density, arXiv:1410.2517 [math.DG] (accessed on 20 July 2017).

[16] O. Röschel, Die Geometrie des Galileischen Raumes, Bericht der Mathematisch-Statistischen Sektion in der Forschungsgesellschaft Joanneum, Bericht Nr. 256, Habilitationsschrift, Leoben, (1984).

[17] Z.M. Sipus, Ruled Weingarten Surfaces in the Galilean Space, Period. Math. Hung. 56 (2) (2008), 213-225.

[18] D.W. Yoon, Weighted Minimal Translation Surfaces in the Galilean Space with Density, Open Math. 15 (2017), 459-466. 